The work of Drs. Ida Macalpine and Richard Hunter with Professor C. Rimington, F.R.S., on tracking the porphyria gene in the royal houses of Hanover, Prussia, and Stuart, was displayed with a wealth of family trees, first editions of rare books, a test-tube of porphyric urine, and, beside it, another containing Alicante wine-which here did provide a remarkably close match. In this exhibit the humanities were indeed joined with the sciences, and it perhaps recaptured something of the times when learned people, whatever their special pursuits, could talk to each other with open enjoyment instead of huddling with a few trusted colleagues over coffee-cups to plot the next step ahead of a rival team. These conversaziones certainly encourage the belief that scientific research can be a civilized as well as a demonic occupation.

\section{Electric Convulsion Therapy}

The mode of action of E.C.T. still remains uncertain in spite of intensive studies with every biophysical, electrophysiological, psychological, and clinical technique available for the study of the functions of mind and brain. ${ }^{12}$ Anaesthetic and relaxant drugs have no effect on the therapeutic value of the induced major seizures, but treatment fails if only partial attacks are induced. This makes it fairly certain that the changes in the central nervous system which accompany a generalized major epileptic seizure are responsible for the therapeutic effect of E.C.T. The electrophysiological, biochemical, and endocrine effects are widespread and may last for hours, even days, after the actual fit and are cumulative with successive convulsions.

Since the therapeutic value of E.C.T. is limited to depression and to a lesser extent mania, it might be thought that the recent intensive studies of the biochemical and physiological aspects of depression could be joined on at some point to theories of the mechanism of E.C.T. In recent years much attention has been paid to mineral metabolism. ${ }^{3}$ In depression, and to even a greater extent in mania, residual sodium, as measured by whole-body studies, is increased. The electrolyte equilibrium is affected by steroid hormones, and adrenal cortical activity has been extensively investigated in pathological emotional states. ${ }^{4}$ It has been known for some time ${ }^{5}$ that E.C.T. affects the output of adrenal cortical steroids. More recently C. G. Clower and C. J. Migeon ${ }^{6}$ have shown some correlation between excretion of 17 -hydroxycorticosteroids and the therapeutic effects of a course of E.C.T. in depression. Urinary 17-O.H.C.S. outputs are raised before treatment in most patients and the levels are reduced by E.C.T. Whole-body studies are, however, of limited value in studying neurochemistry, as the authors point out. D. M. Woodbury ${ }^{7}$ showed that radioactive cortisol entered the cerebral cortex very rapidly and that it led to an increase in

Holmberg, G., Int. Rev. Neurobiol., 1963, 5, 389.

Miller, E., Brit. F. Psychiat., 1967, 113, 301.

Shaw, D. M., Brit. med. F., 1966, 2, 262.

Rubin, R. T., and Mandell, A. J., Amer. F. Psychiat., 1966, 123, 387

Bliss, E. L., Migeon, C. J., Nelson, D. H., Samuels, L. T., and Beurol. Psychiat. (Chic.), 1954, 72, 352 .

Clower, C. G., and Migeon, C. J., fohns Hopk. med. F., 1967, 121, 227.

Woodbury, D. M., Pharmacol. Rev., 1958, 10, 275.

Woodbury, D. M., Pharmacol. Rev., 1958, 10, 275

- Coppen, A., Brit. F. Psychiat., 1965, 111, 11

1 Carney, M. W. P., Roth, M., and Garside, R. F., Brit. ,7. Psychol., 1965, 111, 659.

1 Ottoson, J. O., Acta psychiat. scand., 1960, Suppl. No. 145.

12 Cannicott, S. M., and W.aggoner, R. W., Arch. gen. Psychiat., 1967, 16, 229. intracellular sodium and neuronal excitability. Clearly the changes in the levels of corticosteroids are linked to the abnormalities in electrolytes found by A. Coppen ${ }^{8}$ and others, though the hormonal changes are not invariably found in severe depression. Other biochemical changes have been described in depression. For example, amine metabolism has been a subject of recent research ${ }^{9}$ relating it to the therapeutic effects of monoamine oxidase inhibitors. This series of drugs is often effective in the treatment of depression, though it is not known whether their mode of action is in any way related to that of E.C.T., particularly since their therapeutic effects are not entirely parallel.

It is generally assumed that the site of the important changes in neuronal excitability is somewhere in the thalamic or mid-brain region, though the work of $M$. W. P. Carney and his colleagues ${ }^{10}$ using electroencephalography suggested frontal or thalamo-frontal connexions. This theory could explain the beneficial effect of spontaneous major seizures on some abnormal depressive states among temporal lobe epileptics. It is doubtful whether whole-body biochemical studies can take us much further, but the neuronal interchange of electrolytes and hormones is difficult to investigate even in animals and much more so in humans. Moreover, there is unfortunately no clear animal analogue to the very human disorder of depression.

Clinical and psychological studies of E.C.T. are somewhat disappointing, since the former have been mostly concerned with therapeutic efficacy in purely clinical trials and the latter with describing and attempting to explain the more unsatisfactory side-effects, such as memory loss, that E.C.T. can produce. The work of $\mathrm{J}$. O. Ottosson ${ }^{11}$ on the effects of different current strengths on post-E.C.T. memory loss and studies $^{12}$ of the smaller effects on memory of unilateral administration of the convulsing current suggest that the memory changes are related less to the actual fit and more to the amount of current passed through certain parts of the brain-so that this psychological effect of E.C.T. plays no part in its therapeutic efficacy.

As E.C.T. is nowadays mainly given under an anaesthetic and there is comparatively little discomfort, theories that its efficacy is due to fear, punishment, or regression are generally and rightly discounted. On the other hand, surprisingly little work has been done on the purely affective changes that E.C.T. undeniably produces, which may well be the essential core of its psychological therapeutic efficacy-the changes in thinking and content may be purely secondary. R. T. Rubin and A. J. Mandell ${ }^{4}$ suggested that increased adrenal cortical secretion is most clearly correlated with "loss of ego defence" - that is, the absence of denial, awareness of illness, and suffering with anxiety and depressive affect. The current neuropsychological studies on the physiological basis of affective changes are inconclusive, though the frontal area is still being examined, and interest in the temporal region has shifted deeper into the rhinencephalic area with its intimate connexions to the mid-brain and hypothalamic regions.

As a rough generalization we may therefore say that investigations are beginning to converge on three aspects of possible common mechanisms in depression and E.C.T. Biochemical research is centred on electrolyte and amine metabolism in relation to neuronal excitability; anatomical studies suggest that this excitability cycle may be of prime importance in the mid-brain hypothalamic area; while psychological research suggests that the excitability may be linked to the physiological basis of affect. 